\title{
CAUSAS ASSOCIADAS À MORTE DE MATRIZES SUÍNAS
}

\section{Causes of mortality in swine female}

\author{
VEARICK, G. ${ }^{1}$; MELLAGI, A.P.G ${ }^{1}$; BORTOLOZZO, F.P. ${ }^{1}$; WENTZ, I. ${ }^{1}$; BERNARDI, M.L. ${ }^{2}$
}

${ }^{1}$ Setor Suínos, Faculdade de Veterinária/UFRGS - E-mail: fpbortol@ufrgs.br

${ }^{2}$ Departamento de Zootecnia, Faculdade de Agronomia/UFRGS

\section{RESUMO}

A taxa anual de mortalidade de matrizes suínas varia consideravelmente entre as granjas. A mortalidade, junto com o descarte de matrizes, implica em grandes perdas para 0 produtor. O objetivo deste trabalho foi avaliar as causas de mortalidade de matrizes suínas em uma granja localizada no Centro-oeste brasileiro. Foram realizadas necropsias de 78 matrizes, que morreram ou foram sacrificadas, durante os meses de janeiro e fevereiro de 2006. A taxa de mortalidade observada foi $8,1 \%$, considerada alta em comparação às taxas observadas historicamente nas granjas. As três principais causas de morte foram infecções gênito-urinárias $(30,8 \%)$, seguidas de úlcera gástrica rompida $(14,1 \%)$ e fêmeas sacrificadas $(14,1 \%)$. Problemas de parto e falha cardíaca acometeram $10,3 \%$ e $10,3 \%$ das fêmeas, respectivamente. Em 5,1\% das fêmeas não foi possível definir a causa da morte. Dentre as fêmeas que morreram, 70,5\% apresentavam cistite, $37,2 \%$ pielonefrite e $79,5 \%$ apresentavam algum grau de ulceração no estômago. Das fêmeas que morreram por infecção urinária, 54,2\% tiveram cinco ou mais partos. As fêmeas que morreram por úlcera gástrica tiveram, em média, 1,8 partos, sendo que $90,9 \%$ tiveram no máximo até três partos. O escore de condição corporal das fêmeas sacrificadas foi menor do que o observado nas fêmeas que morreram por outras causas. Foi constatado maior número de mortes nos dias com temperatura superior a $33^{\circ} \mathrm{C}$.

Palavras-Chave: causas de morte, fêmeas suínas, lesões, mortalidade, temperatura.

\section{ABSTRACT}

Annual rate of mortality of sows varies considerably among farms. The increase in mortality, together with culling rate of sows, imply in great losses for the producer. The aim of this work was to evaluate the causes of sows mortality in a farm located in Brazilian Middlewest region. Necropsies of 78 sows were carried out, after their death or euthanasia, during January and February 2006. Mortality rate was $8.1 \%$, considered high if compared to historical rates observed in swine farms. The major causes of death were genito-urinary infections $(30.8 \%)$, followed by hemorrhagic gastric ulcer $(14.1 \%)$ and females euthanatizia (14.1\%). Farrowing problems and cardiac failure occurred in $10.3 \%$ and $10.3 \%$ of dead females, respectively. In $5.1 \%$ of the sows was not possible to determine the cause of death. Among the females that died $70.5 \%$ presented cystitis, $37.2 \%$ pyelonephritis and $79.5 \%$ presented some degree of stomach ulceration. Among females that died by urinary infection, $54.2 \%$ had five or more parities. Females with gastric ulcer had on average of 1.8 parities and $90.9 \%$ of them had less than three parities. The body condition score of females submitted to euthanasia was lower than that observed in dead females due to other reasons. More females died when maximum ambient temperature was above $33^{\circ} \mathrm{C}$.

Palavras-chave: death reasons, lesions, mortality, sows, temperature. 


\section{INTRODUÇÃO}

Há uma grande amplitude na taxa de mortalidade de matrizes ( 0 a $23,5 \%$ ) entre as granjas, devido ao padrão sanitário e de manejo que cada unidade possui (D'allaire et al., 1991; D'allaire et al., 1996; Palomo, 2006). Exceto em casos de surtos, o óbito de matrizes é normalmente negligenciado (Christensen et al., 1995; Abiven et al., 1998). Entretanto, os prejuízos são iminentes e estão relacionados com os custos de reposição da fêmea que morreu, da ração consumida, da leitegada perdida de fêmeas que morrem no período gestacional, além de custos com eventual medicação, mão-de-obra, custo de oportunidade, entre outros (D'allaire et al., 1996; Palomo, 2006). A produtividade do plantel também é afetada, devido à mudança na distribuição da idade do rebanho, aumentando o número de fêmeas jovens com menor nível de produção.

Além disso, a mortalidade de matrizes reflete a saúde geral do plantel e, também, o bem-estar animal. Dentre as principais causas de morte de fêmeas suínas estão as torções de órgãos abdominais, falhas cardíacas, cistites (Chagnon et al., 1991; Abiven et al., 1998; Kirk et al., 2005), eutanásia, problemas de parto e úlcera gástrica (D'allaire et al., 1991; Veestgaard et al., 2006; Palomo, 2006). O objetivo do presente estudo foi identificar as causas associadas à mortalidade de matrizes em uma granja do Centro-Oeste do Brasil que estava apresentando mortalidade acima dos índices aceitáveis, com intuito de discutir estratégias para solucionar o problema.

\section{MATERIAL E MÉTODOS}

O trabalho foi realizado em uma unidade produtora de leitões (UPL) de 12.000 matrizes do centro-oeste brasileiro, no estado de Mato Grosso, que apresentou em 2005 uma taxa de mortalidade de matrizes de $8,1 \%$ (variação mensal entre
6,9 e 9,7\%). Nos meses de janeiro e fevereiro de 2006, foram necropsiadas 78 matrizes de uma mesma genética, que morreram ou foram sacrificadas. As necropsias foram realizadas em um intervalo máximo de 12 horas após o óbito da matriz. Os achados macroscópicos das necropsias foram interpretados segundo as descrições de Sims e Glastonbury (1996) e Sobestiansky e Barcellos (2007). Baseado nesses achados as causa de morte das matrizes foram classificadas em: infecção urinária, úlcera gástrica rompida, problemas de parto, falha cardíaca e outras (enteropatia proliferativa suína, pneumonia, doença de Glässer, eperitrozoonose, septicemia, torção intestinal e torção hepática). No caso de impossibilidade de associar as lesões a uma causa específica que levou ao óbito, foi feita a classificação sem diagnóstico.

Foi avaliado o histórico das fêmeas, incluindo ordem de parto (OP), escore corporal visual (ECV), fase reprodutiva e idade. O ECV foi avaliado antes da realização da necropsia, sendo estabelecida uma pontuação de 1 (caquética) a 5 (obesa), com intervalo de 0,5 ponto, sendo 3,0 considerado ideal. A definição da causa morte das matrizes foi baseada no exame macroscópico.

Durante 38 dias, às 10 horas da manhã, foi registrada a temperatura ambiental das instalações, utilizando termômetros de máxima e mínima, localizados dentro das instalações de gestação e maternidade. No período em que a temperatura ambiental foi aferida, foi avaliado o número de mortes que ocorreram por dia. Para tanto, foram incluídas seis fêmeas que não foram necropsiadas e excluídas as fêmeas sacrificadas, perfazendo um total de 69 matrizes avaliadas frente a essa variável.

O número diário de mortes foi distribuído em 3 classes de temperatura ambiental máxima, da seguinte maneira: 27 a $29,5^{\circ} \mathrm{C}, 30$ a $32,5^{\circ} \mathrm{C}$ e 33 a $34,5^{\circ} \mathrm{C}$. A OP e o ECV observados nas cinco principais 
causas de mortalidade, e o número diário de mortes por classe de temperatura foram analisados pelo procedimento GLM do SAS, sendo as médias comparadas pelo teste de Tukey-Kraemer, em nível de 5\% de significância.

\section{RESULTADOS E DISCUSSÃO}

A taxa de mortalidade média observada na propriedade ao longo do ano anterior $(8,1 \%)$ é considerada alta em comparação àquelas observadas historicamente nas granjas. De acordo com Connor (1989), o nível de mortalidade de matrizes deve ser $<3 \%$ e, caso fique acima de $5 \%$, deve-se tomar medidas de intervenção. Entretanto, corrobora com estudos anteriores que indicam variação de 0 a 23,5\% nas taxas de mortalidade média (D'allaire et al., 1991; D'allaire et al., 1996; Palomo, 2006), embora Straw (1984) sugere uma taxa de $5 \%$ para granjas com mais de 200 matrizes. A taxa de mortalidade está diretamente relacionada com o tamanho do rebanho e, por isso, granjas maiores possuem mortalidade mais elevada do que as granjas de menor porte (Straw, 1984; Christensen et al., 1995; Koketsu, 2000). Isso pode estar relacionado à política de descartes da granja, assim como o maior número de fêmeas por funcionário. $O$ fato de mais de $60 \%$ das mortes estarem concentradas em fêmeas de até 2 partos confirma a observação de Palomo (2006) de que 52\% das mortes de matrizes ocorrem até essa ordem de parição.

De acordo com os dados apresentados na tabela 1 , das 78 fêmeas necropsiadas, $30,8 \%$ morreram por infecção urinária, sendo esta a principal causa de morte diagnosticada. Em seguida ficaram matrizes que morreram em conseqüência de ulceração da porção do quadrilátero esofágico do estômago $(14,1 \%)$ e as fêmeas sacrificadas (14,1\%). Problemas de parto e falha cardíaca representaram 10,3\% cada um. Outras causas de mortalidade, como enteropatia proliferativa suína, pneumonia, doença de Glässer, eperitrozoonose, septicemia, torção intestinal e torção hepática, representaram juntas $15,4 \%$ das mortes.

$\mathrm{O}$ alto percentual de infecções urinárias tem sido relatado freqüentemente como uma das principais causas de morte de matrizes (Kirk et al., 2005; Tarrés et al., 2005). Das fêmeas que morreram por infecção urinária, $54,2 \%$ tiveram cinco ou mais partos, corroborando com dados de trabalho anterior, no qual $64 \%$ dessas matrizes haviam parido cinco ou mais vezes (Chagnon et al., 1991). O ECV médio das matrizes com infecção urinária foi de 3,8 , sendo que $85 \%$ apresentavam ECV maior ou igual a 3,5. Problemas locomotores, insuficiente consumo de água ou baixa qualidade da mesma, má higiene das instalações, freqüência de arraçoamento, entre outros fatores, são importantes na difusão desse problema (Sobestiansky e Barcellos, 2007).

O percentual encontrado de úlcera gástrica rompida pode ser considerado alto quando comparado ao observado em estudos anteriores. Palomo (2006) encontrou um percentual de mortes por úlcera de $6,4 \%$. As fêmeas que morreram por úlcera gástrica tiveram OP média de 1,8 , sendo que $90,9 \%$ estavam com 3 ou menos parições. O ECV médio dessas fêmeas foi de 3,5.

O sacrifício de matrizes é uma importante causa de mortalidade de fêmeas suínas (Stein et al., 1990; Christensen et al., 1995), com índices de até 28,5\%, considerado muito alto. No presente estudo, o sacrifício de fêmeas esteve entre as três principais causas de mortalidade. Altos índices de eutanásia são indicativos de problemas sanitários e de manejo, como falhas na identificação precoce de animais necessitando de tratamento. Segundo Kirk et al. (2005), a maior causa de sacrifício de matrizes está relacionada a problemas locomotores (72\%). Geralmente, as 
Tabela 1 - Causas de mortalidade de fêmeas suínas no Estado do Mato Grosso (2006).

\begin{tabular}{lcccc}
\hline Causa morte & $\mathrm{n}$ & $\%$ & OP & ECV \\
\hline Infecção Urinária & 24 & 30,8 & $4,8 \pm 3,4_{\mathrm{a}}$ & $3,8 \pm 0,6_{\mathrm{a}}$ \\
Úlcera Rompida & 11 & 14,1 & $1,8 \pm 1,7_{\mathrm{b}}$ & $3,5 \pm 0,6_{\mathrm{a}}$ \\
Sacrificadas & 11 & 14,1 & $2,3 \pm 2,5_{\mathrm{ab}}$ & $1,1 \pm 0,2_{\mathrm{b}}$ \\
Problema de parto & 8 & 10,3 & $1,9 \pm 2,8_{\mathrm{ab}}$ & $3,6 \pm 0,7_{\mathrm{a}}$ \\
Falha Cardíaca & 8 & 10,3 & $2,2 \pm 2,9_{\mathrm{ab}}$ & $3,6 \pm 0,5_{\mathrm{a}}$ \\
Outras causas & 12 & 15,4 & $1,0 \pm 1,5_{\mathrm{b}}$ & $3,3 \pm 0,7_{\mathrm{a}}$ \\
Sem Diagnóstico & 4 & 5,1 & $1,7 \pm 1,0_{\mathrm{ab}}$ & $3,4 \pm 0,8_{\mathrm{a}}$ \\
\hline
\end{tabular}

${ }^{1} \mathrm{OP}:$ ordem de parto

${ }^{2}$ ECV: escore corporal visual

${ }^{3} a, b$ letras diferentes indicam diferença significativa $(P<0,05)$

Tabela 2 - Mortalidade de acordo com a fase reprodutiva de matrizes.

\begin{tabular}{|c|c|c|c|c|c|c|c|c|}
\hline \multirow[b]{3}{*}{ Causa Morte } & \multicolumn{8}{|c|}{ FASE REPRODUTIVA } \\
\hline & \multicolumn{2}{|c|}{ IDE } & \multicolumn{2}{|c|}{ GESTAÇÃO } & \multicolumn{2}{|c|}{ MATERNIDADE } & \multicolumn{2}{|c|}{ RECRIA } \\
\hline & $\mathrm{n}$ & $\%$ & $\mathrm{n}$ & $\%$ & $\mathrm{n}$ & $\%$ & $\mathrm{n}$ & $\%$ \\
\hline Infecção Urinária & 6 & 54,6 & 11 & 34,4 & 6 & 20,7 & 1 & 16,7 \\
\hline Úlcera Gástrica & 1 & 9,1 & 2 & 6,2 & 7 & 24,1 & 1 & 16,7 \\
\hline Sacrificada & 1 & 9,1 & 6 & 18,8 & 3 & 10,3 & 1 & 16,7 \\
\hline Problemas de parto & 0 & 0,0 & 1 & 3,1 & 7 & 24,1 & 0 & 0,0 \\
\hline Falha Cardíaca & 2 & 18,2 & 3 & 9,4 & 2 & 6,9 & 1 & 16,7 \\
\hline Sem diagnóstico & 0 & 0,0 & 3 & 9,4 & 1 & 3,5 & 0 & 0,0 \\
\hline Outras & 1 & 9,1 & 6 & 18,8 & 3 & 10,3 & 2 & 33,3 \\
\hline Total & 11 & & 32 & & 29 & & 6 & \\
\hline
\end{tabular}

${ }^{1}$ IDE: Intervalo desmame-estro

matrizes sacrificadas encontram-se muito magras e em decúbito. Sempre que possível, fêmeas em decúbito durante o período gestacional são mantidas na granja até o parto. Porém, elas apresentam dificuldade de ingestão de água e alimento. Muitas vezes, o resultado da gestação dessas matrizes é o abortamento, sendo então sacrificadas. As fêmeas sacrificadas apresentaram, em média, OP de 2,3 e ECV de 1,1 . Todas as fêmeas sacrificadas tinham um ECV igual ou inferior a 1,5, o que comprova a situação crítica dessas fêmeas, em termos de condição corporal. Para evitar o sacrifício, é recomendado realizar manejos diferenciados, oferecendo melhor conforto, como facilitar o acesso à água e à comida e manter a higiene da instalação. Isto permite a manutenção da matriz e, se possível, levá-las até o parto, para que sejam removidas mais tarde, não sendo contabilizadas na taxa de mortalidade.

Os problemas de parto foram responsáveis por $10,3 \%$ das mortes das matrizes. Vestergaard et al. (2006) relataram que as mortes envolvendo 0 parto chegaram a $23 \%$ do total das mortes, em 10 granjas. Já Palomo (2006) relatou que $8,8 \%$ das fêmeas morrem por problemas de parto. Dentre os fatores associados com as complicações do parto estão a inapropriada assistência, a retenção de leitões e fetos macerados, 
síndrome da disgalactia pós-parto, distocia e prolapso de útero ou de vagina. Como os problemas de parto acometeram mais fêmeas jovens (OP média de 2,1), pode-se inferir que foi devido à maior dificuldade em parir, uma vez que animais menores possuem o canal de parto mais estreito. $O$ ECV das matrizes com problemas de parto foi de 3,6. Porém, Christensen et al. (1995) relacionaram problemas de parto a fêmeas com estado corporal acima do normal.

Fêmeas com falhas cardíacas representaram $10,3 \%$ dos resultados das necropsias. Chagnon et al. (1991) demonstraram que as falhas cardíacas representaram $31,4 \%$ das mortes de matrizes suínas, sendo a principal causa de mortalidade. A falha cardíaca acometeu matrizes com ECV médio de 3,6, sendo que $50 \%$ delas apresentavam ECV maior ou igual a 4. Mortes associadas aos meses quentes geralmente estão associadas com falhas cardíacas. Alta temperatura ambiental, parto, obesidade e eventos estressantes predispõem a falhas cardíacas. As matrizes com falha cardíaca tiveram OP média de 1,9. Porém, Chagnon et al. (1991) verificaram que $2 / 3$ das fêmeas que morrem por falha cardíaca tinham, em média, OP de 4,1.

Dentre as fêmeas que foram necropsiadas, 11 estavam no intervalo desmame-estro (14,1\% - média de 6,8 dias após o desmame), 32 na gestação (41,0\% média de 77,7 dias de prenhez), 29 na lactação (37,2\% - média de 8,1 dias de lactação), e 6 estavam na recria (7,7\%). A causa de morte predominante nas fêmeas gestantes e no IDE foi infecção urinária, podendo estar relacionada ao menor cuidado higiênico nestas instalações e menor consumo de água. Já as fêmeas na maternidade morreram mais por úlcera gástrica e problemas de parto seguido de infecções urinárias (Tabela 2).

A interrupção de arraçoamento em leitoas transferidas para a linha de cobertura e próximo ao período do parto, pode ser um fator para ocorrência de úlcera gástrica, podendo levar a uma quebra da homeostase do $\mathrm{pH}$ estomacal, induzindo as lesões de úlcera na porção esofágica do estômago. A úlcera gástrica também pode estar relacionada à diminuição do consumo alimentar, comum nesta fase, e à temperatura do ambiente da maternidade, que agrava a queda de consumo (Friendship, 1999).

Os achados de necropsia de acordo com a causa de morte das fêmeas estão apresentados na tabela 3 . Independemente da causa de mortalidade, $83,3 \%$ das fêmeas necropsiadas apresentaram ulceração da porção esofágica do estômago e 34,6\% delas com grau 4 de ulceração. Dados semelhantes foram encontrados no exame de fêmeas descartadas, das quais $60 \%$ dos estômagos estavam com lesões e 10 a $15 \%$ com ulceração (Friendship et al., 1999). Freqüentemente, o estômago das matrizes possui severas e extensivas áreas de tecido cicatricial indicando lesões prévias de ulceração severa.

Das fêmeas que morreram por infecção urinária, todas apresentaram cistite e 54,2\% apresentaram pielonefrite. A associação freqüente encontrada entre cistite e pielonefrite também já foi relatada (Tarrés et al., 2005; Sanz et al, 2007), sendo a última responsável pela mortalidade. Achados de cistite e pielonefrite em fêmeas que morreram por problemas de parto corroboram com Sanz et al. (2007).

Das fêmeas sacrificadas, $63,6 \%$ apresentaram pneumonia e $45,5 \%$ pleurisia. Lesões de artrite representaram $54,5 \%$ e $18,2 \%$ dessas matrizes apresentaram epifisiólise. Oito das 11 fêmeas apresentaram algum grau de ulceração estomacal. Achados semelhantes foram encontrados por Kirk et al. (2005), os quais constataram presença de lesões locomotoras e de úlcera, em fêmeas sacrificadas.

O número diário de mortes de acordo com a classe de temperatura 
Tabela 3 - Percentual de achados de necropsia de acordo com a causa de mortalidade de matrizes.

\begin{tabular}{lccccc}
\hline Lesões & $\begin{array}{c}\text { Infecção } \\
\text { Urinária }\end{array}$ & Sacrificada & $\begin{array}{c}\text { Úlcera } \\
\text { Gástrica }\end{array}$ & $\begin{array}{c}\text { Problema de } \\
\text { Parto }\end{array}$ & $\begin{array}{c}\text { Falha } \\
\text { Cardíaca }\end{array}$ \\
\hline Cistite & 100,0 & 36,4 & 54,5 & 77,8 & 62,5 \\
Pielonefrite & 54,2 & 9,1 & 54,5 & 22,2 & 37,5 \\
Depósito Mineral na Pelve & 29,2 & 36,4 & 9,1 & 33,3 & 12,5 \\
Cisto Renal & 16,7 & 27,3 & 9,1 & 11,1 & 0,0 \\
Pneumonia & 12,5 & 63,6 & 18,2 & 22,2 & 0,0 \\
Pleurisia & 16,7 & 45,5 & 27,3 & 33,3 & 12,5 \\
Hidrotórax & 50,0 & 0,0 & 18,2 & 33,3 & 37,5 \\
Edema Pulmonar & 87,5 & 0,0 & 72,7 & 33,3 & 62,5 \\
Úlcera Gástrica & 79,2 & 72,7 & 100,0 & 88,9 & 50,0 \\
Ascite & 70,8 & 27,3 & 72,7 & 77,8 & 87,5 \\
Hidropericárdio & 87,5 & 27,3 & 81,8 & 44,4 & 75,0 \\
Congestão Hepática & 54,2 & 9,1 & 9,1 & 22,2 & 37,5 \\
Artrite & 8,3 & 54,5 & 0,0 & 11,1 & 12,5 \\
\hline
\end{tabular}

ambiental é apresentado na Tabela 4. Houve tendência de maior número diário de mortes, no grupo de dias com temperatura máxima a partir de $33^{\circ} \mathrm{C}(\mathrm{P}<0,08)$ em comparação ao grupo de dias com temperatura abaixo de $30^{\circ} \mathrm{C}$. A observação de maior número de mortes, nos dias com temperatura máxima a partir de $33^{\circ} \mathrm{C}$, reforça os relatos de que períodos de temperaturas elevadas estão associados com altas taxas de mortalidade de matrizes suínas (Veestgaard et al., 2006; Sanz et al., 2007).

Em situações de alto percentual de mortalidade de matrizes, as principais causas devem ser identificadas e associadas à fase de produção, idade e condição física das fêmeas. Com isso, ações direcionadas por fase de produção podem ser implementadas, assim como tentativas para minimizar as perdas por estresse térmico e para melhorar as condições de fêmeas candidatas ao sacrifício. Falhas de manejo também estão associadas à alta taxa de mortalidade e, por isso, medidas preventivas podem ser adotadas para aumentar a longevidade das fêmeas.

Tabela 4 - Número de mortes por dia de acordo com a classe de temperatura máxima.

Classe de temperatura Dias Mortes № mortes/dia

\begin{tabular}{cccc}
27 a $29,5^{\circ} \mathrm{C}$ & 10 & 10 & $1,0_{\mathrm{a}}$ \\
30 a $32,5^{\circ} \mathrm{C}$ & 18 & 30 & $1,7_{\mathrm{a}}$ \\
33 a $34,5^{\circ} \mathrm{C}$ & 10 & 29 & $2,9^{\mathrm{b}}$ \\
\hline
\end{tabular}

${ }^{1} a, b$ letras diferentes indicam diferença significativa $(P<0,10)$

\section{CONCLUSÃO}

As principais causas de mortalidade são infecções urinárias e úlcera gástrica rompida. A morte por infecção urinária é mais freqüente em fêmeas mais velhas ao passo que a úlcera gástrica ocorre mais em fêmeas com menos de três parições. Fêmeas sacrificadas são as que possuem baixo escore de condição corporal. $O$ número diário de mortes aumentou em três vezes nos dias mais quentes. 


\section{REFERÊNCIAS}

ABIVEN, N.; SEEGERS, H.; BEAUDEAU, F.; LAVAL, A.; FOURICHON, C. Risk factors for high sow mortality in French swine herds. Preventive Veterinary Medicine, v.33, n.1-4, p.109-119, 1998.

CHAGNON, M.; D'ALLAIRE, S.; DROLET, R. A prospective study of sow mortality in breeding herds. Canadian Journal of Veterinary Research, v.55, n.2, p.180-184, 1991.

CHRISTENSEN, G.; VRAA-ANDERSEN, L.; MOUSING, J. Causes of mortality among sows in Danish pig herds. The Veterinary Record, v.137,n.16, p.395-399, 1995.

CONNOR, J. Reproductive problems in swine breeding herds: Making the field diagnosis. Journal of Veterinary Medicine, v.83, n. 3, p.318-327, 1989.

D'ALLAIRE, S.; DROLET, R.; BRODEUR, D. Sow mortality associated with high ambient temperatures. Canadian Veterinary Journal, v.37, n.4, p.237-239, 1996.

D'ALLAIRE, S.; DROLET, R.; CHAGNON, M. The causes of sow mortality: A retrospective study. Canadian Veterinary Journal, v.32, n.4, p.241-243, 1991.

FRIENDSHIP, R. Gastric Ulcer. In: STRAW, B; D'ALLAIRE, S.; MENGELING, W. L.; TAYLOR, D. J. Diseases of swine. 8th ed. lowa: lowa university press, 1999, p. 685-694.

KIRK, R.K.; SVENSMARKM, B.; ELLEGAARD, L.P.; JENSEN, H.E. Locomotive disorders associated with sow mortality in Danish pig herds. Journal of Veterinary Medicine, v.52, n.8, p.423-428, 2005.

KOKETSU, Y. Retrospective analysis of trends and production factors associated with sow mortality on swine-breeding farms in USA. Preventive Veterinary Medicine, v.46, n.4, p.249-256, 2000.

PALOMO, A. Analysis of sow mortality among breeding sows in Spanish pig herds. In: ALLEN D. LEMAN SWINE CONFERENCE. 2006, Minnesota, Proceedings... Minnesota: College of Veterinary Medicine, University of Minnesota, 2006, v.33, 3p.

SANZ, M.; ROBERTS, J.D.; PERFURMO, C.J. Assessment of sows mortality in a large herd. Journal of Swine Health and Production. v.15, n.1, p.30-36. 2007.

STEIN, T.E.; DIJKHUIZEN, A.; D'ALLAIRE, S.; MORRIS, R.S. Sow culling and mortality in commercial swine breeding herds. Preventive Veterinary Medicine, v.9,n.2, p.85-94, 1990.
STRAW, B. Causes and Control of sow losses. Modern veterinary practices, USA, v.65, n.5, p. 349-353, 1984.

SOBESTIANSKY, J; BARCELLOS, D.E.S.N. Doenças dos Suínos. Goiânia: Cânone Editorial, 2007, 770p.

SIMS, L.D.; GLASTONBURY, J.R.W. Pathology of the pig. Victoria-Australia: The pig researche and development corporation, 456p., 1996.

TARRÉS, J.; TIBAU, J.; PIEDRAFITA, J.; FÀBREGA, E.; REIXACH, J. Factors affecting longevity in maternal Duroc swine lines. Livestock Science, v.100, p.121-131, 2005.

VEETERGAARD, K.; BAEKBO, P.; SVENSMARK, B. Sow mortality and causes for culling of sows in Danish pig herds. In: INTERNATIONAL PIG VETERINARY SOCIETY CONGRESS, IXX, 2006, Copenhagen, Proceedings... Copenhagen: IPVS, 2006, p.255. 